\title{
Exaggerated circulating Th-1 cytokine response in early rheumatoid arthritis patients with nodules
}

Hodkinson $\mathrm{B}^{1}$, Meyer PWA ${ }^{2}$, Musenge $\mathrm{E}^{3}$, Ally $\mathrm{M}^{4}$, Anderson $\mathrm{R}^{2}$ and Tikly $\mathrm{M}^{1}$

${ }^{1}$ Division of Rheumatology, Department of Medicine, Chris Hani Baragwanath Hospital, University of the Witwatersrand, Johannesburg, South Africa, ${ }^{2}$ Medical Research Council Unit for Inflammation and Immunity, Department of Immunology, Faculty of Health Sciences, University of Pretoria and National Health laboratory Service, Tshwane Academic Division, Pretoria , ${ }^{3}$ Biostatistics and Epidemiology Division, School of Public Health, Faculty of Health Sciences, University of the Witwatersrand, Johannesburg, ${ }^{4}$ Department Internal Medicine, University of Pretoria, Pretoria

Correspondence to: Bridget Hodkinson MBBCh, FCP (SA)

Division of Rheumatology,

Department of Medicine

Chris Hani Baragwanath Hospital

PO Box Bertsham 2013

South Africa

Telephone: +27 119339377

Facsimile: +27 119388738

Email: drbridget@gmail.com 


\section{Abstract}

Background: Immunohistochemical studies of the rheumatoid nodule (RN) suggest it is a Th1 granuloma, with focal vasculitis, yet the pathogenesis remains unclear and little is known about circulating cytokines in these patients. Objective: We studied circulating cytokines in DMARD-naïve RA patients to investigate associations with subcutaneous RN. Methods: 149 DMARD-naïve adults with early RA (symptom duration $\leq 2$ years) were assessed using the Simplified Disease Activity Index (SDAI), and hand and feet radiographs were scored using the modified Larsen method. Circulating cytokines and growth factors representative of T- helper cell 1(Th1) and Th2 cell, macrophages, and fibroblasts were measured using the Bio-Plex® suspension array system. Results: Of 149 patients, 34 (22.8\%) had subcutaneous RN, and these patients had more severe disease with higher mean swollen joint counts $(p=0.02)$, SDAI $(p=0.04)$ and modified Larsen scores $(p=0.004)$. There were no differences in Rheumatoid Factor or anti-cyclic citrullinated peptide antibody positivity between patients with $\mathrm{RN}$ and those without $\mathrm{RN}$. Patients with $\mathrm{RN}$ showed significantly higher levels of circulating IL-12 ( $p=0.02)$, IL-2 $(p=0.048)$, and VEGF $(p=0.033)$ levels, with a trend towards higher levels of IL-7 $(p=0.056)$, IFN- $\gamma(p=0.059)$ and IL-8 $(p=0.074)$ compared to those without RN. Conclusions: DMARD-naïve early RA patients with $\mathrm{RN}$ had more severe disease than those without RN, and showed an exaggerated circulating Th1 and macrophage cytokine profile.

Keywords: Rheumatoid nodules, extra-articular disease, circulating cytokines, VEGF 
Rheumatoid nodules $(\mathrm{RN})$ are the most common extra-articular feature of rheumatoid arthritis (RA), and are found most commonly in subcutaneous tissues at the site of recurrent mechanical irritation. Nodules are a feature of established RA, occurring in $30-40 \%$ of patients, with an average disease duration of eleven years (1). Joint inflammation and RN do not run the same clinical course: patients with low disease activity can still develop RN, and an increase in nodule size or formation of new nodules is well described with therapy that suppresses synovitis, such as methotrexate and anti-TNF drugs.

The RN has been described as "the most characteristic histopathological lesion in RA" and as such perhaps deserves more attention(2). Histopathologically, the RN is an immune granuloma consisting of a central area of necrosis, surrounded by a palisade of macrophages and fibroblasts, and a peripheral vascular area containing $T$ lymphocytes and macrophages. A number of studies have drawn comparisons between $\mathrm{RN}$ and rheumatoid synovium, describing similarities between the tissues with respect to cellular components, immunohistochemical features and cytokine production $(3,4)$. There are also important differences including the absence of $B$ lymphocytes and lymphoid follicles in the nodule, variations in expression of cell adhesion molecules and in cytokine gene expression(5).

There is increasing evidence of endothelial dysfunction and angiogenesis in rheumatoid synovium. One marker of angiogenesis is VEGF, and levels correlate with disease activity and radiographic progression(6). A recent study demonstrated higher VEGF levels in patients with extra-articular involvement and the authors concluded that VEGF may be a marker systemic of rather than joint inflammation in RA(7). Recently, IL-8 has been shown to play an important role in angiogenesis(8).

Better understanding of the cytokines present in serum of patients with RN might offer clues to the immunopathogenesis of RN and possibly other extra-articular complications of RA. To our knowledge there are no published studies on the relationship of circulating cytokines with RN. We therefore undertook a cross-sectional study of early RA patients who were disease modifying anti-rheumatic drug (DMARD)naïve to determine the association of $\mathrm{RN}$ with disease activity, radiographic damage, 
autoantibody status, the presence of the shared epitope (SE), and circulating cytokines. This study was approved by the University of the Witwatersrand Committee for Research on Human Subjects.

\section{Patients and Methods}

\subsection{Patients}

149 early RA (defined as disease duration $\leq 2$ years) adults who met the American College of Rheumatology classification criteria for RA and were DMARD naïve were studied. Clinical assessments (Table 1) included a smoking history, the presence of subcutaneous RN, the Simplified Disease Activity Index (SDAI) and the health assessment questionnaire disability index (HAQ-DI). A chest $x$-ray (CXR) was performed and radiographs of hands and feet were scored using the modified Larsen score.

\subsection{Auto-antibody and cytokine assays}

Venous blood $(30 \mathrm{ml})$ was collected in endotoxin-free, silicone-coated vacutainers and allowed to coagulate at room temperature, followed by centrifugation (3000 rpm for 10 minutes). The serum was removed and aliquoted, and stored at minus $20 \circ \mathrm{C}$ until performance of the various assays described below. Rheumatoid factor (RF) was assayed by nephelometry (Siemens Healthcare Diagnostics,BN Prospec Nephelometer, Newark, USA) and anticyclic citrullinated peptide (aCCP) antibodies were measured by a second generation immunofluorometric procedure using the Immunocap 250 system (Phadia AB, Uppsala, Sweden). Values of $15 \mathrm{IU} / \mathrm{ml}$ and $10 \mathrm{U} / \mathrm{ml}$ respectively were considered positive. Serum IL-1b, IL-1Ra, IL-2, IL-4, IL-6, IL-7, IL-8, IL-10, IL-12, IFN- $\gamma$, TNF, G-CSF, GM-CSF, CCL2, CCL4, and VEGF were measured using the Bio-Plex® suspension array system (Bio-Rad Laboratories Inc, Hercules, CA, USA) which utilizes Luminex® XMAP'M multiplex technology. The system uses an array of beads in liquid suspension, each containing different ratios of two spectrally distinct fluorophores, thereby assigning a unique spectral identity. The beads are conjugated with a monoclonal antibody specific for a target proteinand incubated with the serum sample ( $1 / 4$ dilutions), washed, followed by addition of a biotinylated detection antibody, washed 
again, and finally incubated with streptavidin-phycoerythrin. A wide range of standards $(0.38-91756.00 \mathrm{pg} / \mathrm{ml})$ were used to enable quantitation of the individual cytokines using a Bio-Plex array reader with a dual laser detector and real time digital signal processing.

\subsection{Assessment for the Shared Epitope}

Genomic DNA was analysed for the presence of the SE using high-resolution rSSO PCR typing of the RAA amino acid motif at positions 72 to 74 of the third hypervariable region of the different human leucocyte antigen HLA-DRB1 allele, as described previously(9).

\subsection{Statistical analysis}

The Mann-Whitney test was applied to compare continuous variables, and in the case of categorical variables, the Pearson's Chi-Square test, or where indicated, the 2tailed Fishers' Exact test was used. Log transformation was performed on cytokine levels to correct for non-normal distribution. Because the higher mean SDAI in the RN group compared to the non-RN group may explain the differences in cytokine levels, we further analysed our data adjusting for SDAI. As our dependent variable was binary (RN vs non- RN) we employed multiple logistic regression to determine how each of the cytokines changed when we introduced SDAl in the model. Stata 10 software (StataCorp, USA) was used. A p-value of $<0.05$ was considered significant, and $p$ values of $<0.1$ were considered to show a "trend" towards significance.

\section{Results}

\subsection{Clinical features}

The majority of patients were female (81\%), Black Africans (93\%) with mean symptom duration of 11.6 months. A high percentage of patients were RF and aCCP antibody positive (89 and $85 \%$ respectively), and the majority (93\%) carried the SE. 
Subcutaneous RN were observed in 34 patients (22.8\%), and all of these occurred on the extensor surface of the forearm. No patients had nodules on CXR. As shown in Table 1, patients with RN had features of more severe disease with higher mean swollen joint counts, SDAl and modified Larsen scores compared to those without $\mathrm{RN}$. A higher percentage of males were found in the RN group but this did not reach statistical significance, and smokers were no more likely to have RN than non-smokers.

Table 1: Clinical, radiographic and autoantibody features of 149 early RA patients with and without rheumatoid nodules (RN)

\begin{tabular}{llll}
\hline & \multicolumn{1}{c}{$\begin{array}{c}\mathrm{RN} \\
(\mathrm{n}=34)\end{array}$} & \multicolumn{1}{c}{$\begin{array}{c}\text { no RN } \\
(\mathrm{n}=115)\end{array}$} & \multicolumn{1}{c}{ p value } \\
\hline Black Africans (\%) & $32(94.1)$ & $107(93.0)$ & $\mathrm{ns}$ \\
Male (\%) & $10(29.4)$ & $19(16.5)$ & $\mathrm{ns}$ \\
Age (years)- mean (SD) & $46.2(13.9)$ & $46.8(12.4)$ & $\mathrm{ns}$ \\
Duration (months)- mean (SD) & $13.0(7.67)$ & $11.2(7.0)$ & $\mathrm{ns}$ \\
Ever smoked (\%) & $7(20.6)$ & $25(21.7)$ & $\mathrm{ns}$ \\
Swollen joint count - mean (SD) & $16.1(7.6)$ & $12.5(7.7)$ & 0.02 \\
CRP (mg/l))- mean (SD) & $40.7(38.6)$ & $27.5(37.5)$ & 0.07 \\
Simplified Disease Activity Index - mean (SD) & $44.7(14.8)$ & $38.5(16.5)$ & 0.04 \\
Health Assessment Questionnaire- mean (SD) & $1.9(0.8)$ & $1.7(0.7)$ & $\mathrm{ns}$ \\
modified Larsen Score- mean (SD) & $28.3(17.0)$ & $20.7(10.7)$ & 0.004 \\
Erosive disease (\%) & $20(58.8)$ & $59(51.3)$ & $\mathrm{ns}$ \\
RF positive (\%) & $30(88.2)$ & $101(87.8)$ & $\mathrm{ns}$ \\
aCCP positive (\%) & $28(82.4)$ & $87(75.7)$ & $\mathrm{ns}$ \\
\hline
\end{tabular}

SD: standard deviation; RF: Rheumatoid Factor; aCCP: anticyclic citrullinated peptide; CRP: $C$ reactive protein; ns: not significant 
Table 2: Significant differences in cytokine and growth factor levels of RA patients with rheumatoid nodules (RN) compared to those without nodules

\begin{tabular}{|c|c|c|c|c|c|c|}
\hline & \multicolumn{2}{|c|}{ Median (range) } & \multicolumn{2}{|c|}{$\begin{array}{l}\text { Median (range) for log- } \\
\text { transformed }\end{array}$} & \multirow[t]{2}{*}{$\begin{array}{c}\mathrm{p} \\
\text { value* }\end{array}$} & \multirow{2}{*}{$\begin{array}{l}\mathrm{p} \text { value } \\
\text { adjusted } \\
\text { for SDA }\end{array}$} \\
\hline & $\begin{array}{c}\mathrm{RN} \\
\mathrm{n}=34\end{array}$ & $\begin{array}{l}\text { no RN } \\
n=115\end{array}$ & $\begin{array}{c}\mathrm{RN} \\
\mathrm{n}=34\end{array}$ & $\begin{array}{l}\text { no RN } \\
n=115\end{array}$ & & \\
\hline $\mathrm{IL}-1 \mathrm{~b}$ & $7.6(0.1-191.3)$ & $6.2(1.0-242.3)$ & $2.0(-2.3-5.3)$ & $1.8(0.1-5.5)$ & $\mathrm{ns}$ & ns \\
\hline IL-1Ra & $91.6(.-3308.7)$ & $89.6(0-4838.2)$ & $5.1(3.2-8.1)$ & $4.7(1.1-8.5)$ & ns & ns \\
\hline IL-2 & $6.5(0.0-515.8)$ & $0.0(0.0-1319.9)$ & $1.9(-6.9-6.2)$ & $-6.9(-6.9-7.2)$ & 0.04 & 0.048 \\
\hline IL-4 & $89.4(0.0-4996.0)$ & $59.2(0.0-6825.1)$ & $4.5(-6.9-8.5)$ & $3.0(-6.9-8.8)$ & ns & ns \\
\hline IL-6 & $37.7(0-324.6)$ & $26.1(0-1077.7)$ & $37(-13-58)$ & $3.3(0.9-7.0)$ & ns & ns \\
\hline IL-7 & $89.4(0.0-4996.0)$ & $19.2(0.0-6825.1)$ & $4.5(-6.9-8.5)$ & $3.0(-6.9-8.8)$ & 0.002 & 0.056 \\
\hline IL-8 & $12.7(2.2-132.4)$ & $8.7(0.0-572.6)$ & $2.5(0.8-4.9)$ & $2.2(-6.9-6.4)$ & 0.038 & 0.074 \\
\hline $\mathrm{IL}-10$ & $13.2(0-472.1)$ & $13.0(0-1172.1)$ & $2.6(-0.4-6.2)$ & $2.6(0.3-7.1)$ & ns & ns \\
\hline IL-12 & $23.5(1.3-3557.8)$ & $11.7(0.6-3104.6)$ & $3.2(0.3-8.2)$ & $2.5(-0.5-8.0)$ & 0.02 & 0.016 \\
\hline $\mathrm{IL}-17$ & $0(0-228.7)$ & $0(0-38.6)$ & $2.1(-1.1-5.4)$ & $2.0(-0.2-37)$ & ns & ns \\
\hline IFN-Y & $110.2(0.00-11151.7)$ & $56.4(0.0-10922.2)$ & $7.3(1.2-9.3)$ & $4.7(-6.9-9.3)$ & 0.25 & 0.059 \\
\hline TNF & $19.4(29-1364.6)$ & $4.2(0.6-2951.7$ & $3.0(1.1-7.2)$ & $2.7(-0.6-8.0)$ & ns & ns \\
\hline G-CSF & $17.0(0-4817.4)$ & $15.5(0-8764.2)$ & $4.7(0-3726.2)$ & $2.9(-1.7-9.1)$ & ns & ns \\
\hline GM-CSF & $3.4(0-1363.6)$ & $1.1(0-3726.2)$ & $4.4(0-1363.6)$ & $3.6(-2.3-8.2)$ & ns & ns \\
\hline VEGF & $407.9(15.0-2886.4)$ & $128.0(0.0-4503.2)$ & $6.0(2.7-7.9)$ & $4.9(-6.9-8.4)$ & 0.011 & 0.033 \\
\hline CCL2 & $64.2(0-237.3)$ & $53.3(0-417.3)$ & $4.5(1.6-5.5)$ & $4.2(1.4-6.0)$ & ns & ns \\
\hline CCL4 & $129.2(31.9-301.8)$ & $107.6(19.8-643.5)$ & $4.9(3.5-5.7)$ & $4.7(2.3-6.5)$ & ns & ns \\
\hline $\begin{array}{l}\text { IL12:IL-4 } \\
\text { ratio }\end{array}$ & $4.2(0.8-123.7)$ & $9.5(0.6-2503.7)$ & & & 0.08 & 0.15 \\
\hline $\begin{array}{l}\text { IL12:IL10 } \\
\text { ratio }\end{array}$ & $3.2(0.4-25.2)$ & $2.6(0.2-17.3)$ & & & 0.036 & 0.051 \\
\hline
\end{tabular}

Data are presented as $\rho g / m l$; SDAl: simplified disease activity index; ns: not significant ${ }^{*} p$ value for log transformed cytokine levels 


\subsection{Circulating cytokines}

Compared to patients without RN, patients with RN had significantly higher Thelper cell 1(Th1) cytokine levels. As shown in Table 2, IL-12 and IL-2 were higher amongst RN patients even after correction for the SDAI, and there was a trend towards higher levels of IL-7 and IFN- $\gamma$. The Th1/Th2 cytokine ratio of IL-12 to IL-10, but not IL12 to IL-4, was significantly higher in the RN group. In addition, VEGF levels were significantly elevated in the RN group, with higher levels of IL-8 that did not reach significance after correction for the SDAl. There were no significant differences between the two groups in the levels of IL-1b and TNF, Th2 derived cytokines, nor any other cytokine or growth factor measured.

The occurrence of RF and aCCP antibodies was similar in the RN and the non$\mathrm{RN}$ patients. There were no significant differences in the frequency of the SE between the RN and non-RN patients ( $56.0 \%$ vs $54.7 \%$ were homozygous for the SE allele, and $32.0 \%$ vs $38.9 \%$ heterozygous).

\section{Discussion}

In this study of early RA patients, we observed increased serum levels of Th1 and macrophage derived cytokines in the subgroup of patients with $\mathrm{RN}$, independent of disease activity. These findings are consistent with immunohistochemical studies of RN describing a lymphocytic and macrophage infiltrate, with cytokines IFN- $\gamma$, IL-2, TNF and IL1-B extracted from RN (4). Furthermore, levels of VEGF and IL-8 were significantly higher in patients with RN. Elevation of these pro-angiogenic factors might imply that angiogenesis is a prominent feature in RN, in keeping with early and recent descriptions of vascular proliferation in $\mathrm{RN}(10,11)$. However, it could be argued that the circulating VEGF and IL-8 levels are a marker of the overall increased angiogenesis in active RA synovitis (12), rather than indirect evidence of angiogenesis in RN.

The pathogenesis of the RN remains unclear, but local vasculitis has been suggested(13), and our findings supporting a T-cell driven process are compatible with 
this. Possibilities include sensitization of vascular endothelium by circulating cytokines such as TNF and IFN- $\gamma$, as well as CRP. This may result in hyper-responsiveness to minor mechanical trauma to pressure points favouring adhesion of monocytes, localized activation and differentiation of these cells, generation of $T$ cell chemoattractants and activation of these cells by monocyte/macrophage/fibroblast cytokines (IL-7, IL-12) in either the absence or presence of citrullinated autoantigens. Citrullinated proteins have been identified in RN, raising the question of the role of aCCP antibodies in the pathogenesis of the $\mathrm{RN}(14)$.

In this study, circulating IL-7 levels were significantly elevated amongst RN patients. Previously, we and others have shown RA patients to have higher IL-7 levels compared to healthy controls $(15,16)$, although reduced IL-7 levels in RA patients compared to healthy controls or osteoarthritis patients have been reported elsewhere(17).

The black South African RA population has a high frequency of the SE allele, and this is borne out in this study where $93 \%$ of patients carried the SE. We have previously shown an association between the SE and aCCP antibodies, with higher disease activity scores and higher levels of circulating cytokines occurring in these patients(9), perhaps suggesting a predilection to more severe disease. However, in the present study the SE was not a risk for RN. This is in keeping with a large meta-analyses that shows no significant association between SE and nodules(1). Others have reported an association between the SE and RN, and in particular the SE-containing alleles DRB1*0401(18). The low frequency of the SE and of HLA DRB1*0401 in AfroAmericans and Africans has been suggested as an explanation of the low incidence of $\mathrm{RN}$ in these populations(19), but seemingly this is not the case in Southern Africans.

Nearly a quarter of patients in this cohort had RN, and this is higher than the $10 \%$ prevalence described elsewhere in patients with early disease(20). As in other studies, $\mathrm{RN}$ was associated with higher disease activity and radiological damage scores(20). Males have a higher risk of $\operatorname{RN}(1,21)$, however in the present study although more males than females had RN, this did not reach statistical significance. Smoking was not 
associated with RN in the present study, whereas others have reported a strong association(21). The small size of our cohort is one possible explanation.

The chief limitation of this study was that we did not study the relationship between circulating cytokines and the immunohistochemistry of RN. In addition, we were unable to assess the IL-23/IL-17 pathway, as neither IL-21 nor IL-23 was available on the cytokine bead array system at the time of the study. A major strength of this study is that all patients had early disease and were DMARD-naïve; hence therapy was not a confounder in cytokine production or RN formation.

In conclusion, DMARD-naïve RA patients with RN had severe disease, with an exaggerated Th1 and macrophage cytokine profile. Further work is needed to better define the role of these cytokines and IL-17 pathway in the pathogenesis of RN by investigating their relationship with histopathology and immunohistochemistry changes of RN.

\section{Conflict of interest statement}

The authors declare no conflicts of interest.

\section{Acknowledgements}

This work was supported by the Connective Tissue Diseases Research Fund, University of the Witwatersrand and the Medical Research Council of South Africa.

\section{References}

1. Gorman JD, David-Vaudey E, Pai M, Lum RF, Criswell LA. Lack of association of the HLA-DRB1 shared epitope with rheumatoid nodules: an individual patient data meta-analysis of 3,272 Caucasian patients with rheumatoid arthritis. Arthritis Rheum 2004;50:753-62.

2. Highton J, Hessian PA, Stamp L. The Rheumatoid nodule: peripheral or central to rheumatoid arthritis? Rheumatology (Oxford) 2007;46:1385-7.

3. Athanasou NA, Quinn J, Woods CG, McGee JO. Immunohistology of rheumatoid nodules and rheumatoid synovium. Ann Rheum Dis 1988;47:398-403.

4. Hessian PA, Highton J, Kean A, Sun CK, Chin M. Cytokine profile of the rheumatoid nodule suggests that it is a Th1 granuloma. Arthritis Rheum 2003;48:334-8. 
5. Stamp LK, Easson A, Lehnigk U, Highton J, Hessian PA. Different T cell subsets in the nodule and synovial membrane: absence of interleukin-17A in rheumatoid nodules. Arthritis Rheum 2008;58:1601-8.

6. Ballara S, Taylor PC, Reusch P, Marme D, Feldmann M, Maini RN, et al. Raised serum vascular endothelial growth factor levels are associated with destructive change in inflammatory arthritis. Arthritis Rheum 2001;44:2055-64.

7. Kuryliszyn-Moskal A, Klimiuk PA, Sierakowski S, Ciolkiewicz M. A study on vascular endothelial growth factor and endothelin-1 in patients with extra-articular involvement of rheumatoid arthritis. Clin Rheumatol 2006;25:314-9.

8. Li A, Dubey S, Varney ML, Dave BJ, Singh RK. IL-8 directly enhanced endothelial cell survival, proliferation, and matrix metalloproteinases production and regulated angiogenesis. $\mathrm{J}$ Immunol 2003;170:3369-76.

9. Meyer PW, Hodkinson B, Ally M, Musenge E, Wadee AA, Fickl H, et al. HLA-DRB1 shared epitope genotyping using the revised classification and its association with circulating autoantibodies, acute phase reactants, cytokines and clinical indices of disease activity in a cohort of South African rheumatoid arthritis patients. Arthritis Res Ther;13:R160.

10. Ziff M. The rheumatoid nodule. Arthritis Rheum 1990;33:761-7.

11. Baeten D, De Keyser F, Veys EM, Theate Y, Houssiau FA, Durez P. Tumour necrosis factor alpha independent disease mechanisms in rheumatoid arthritis: a histopathological study on the effect of infliximab on rheumatoid nodules. Ann Rheum Dis 2004;63:489-93.

12. Tarner IH, Harle P, Muller-Ladner U, Gay RE, Gay S. The different stages of synovitis: acute vs chronic, early vs late and non-erosive vs erosive. Best Pract Res Clin Rheumatol 2005;19:19-35.

13. Mellbye OJ, Forre O, Mollnes TE, Kvarnes L. Immunopathology of subcutaneous rheumatoid nodules. Ann Rheum Dis 1991;50:909-12.

14. Bongartz T, Cantaert T, Atkins SR, Harle P, Myers JL, Turesson C, et al. Citrullination in extra-articular manifestations of rheumatoid arthritis. Rheumatology (Oxford) 2007;46:70-5.

15. Meyer PW, Hodkinson B, Ally M, Musenge E, Wadee AA, Fickl H, et al. Circulating cytokine profiles and their relationships with autoantibodies, acute phase reactants, and disease activity in patients with rheumatoid arthritis. Mediators Inflamm;2010:158514.

16. Churchman SM, Ponchel F. Interleukin-7 in rheumatoid arthritis. Rheumatology (Oxford) 2008;47:753-9.

17. Ponchel F, Verburg RJ, Bingham SJ, Brown AK, Moore J, Protheroe A, et al. Interleukin7 deficiency in rheumatoid arthritis: consequences for therapy-induced lymphopenia. Arthritis Res Ther 2005;7:R80-92.

18. Weyand CM, Xie C, Goronzy JJ. Homozygosity for the HLA-DRB1 allele selects for extraarticular manifestations in rheumatoid arthritis. J Clin Invest 1992;89:2033-9.

19. Del Rincon I, Battafarano DF, Arroyo RA, Murphy FT, Fischbach M, Escalante A. Ethnic variation in the clinical manifestations of rheumatoid arthritis: role of HLA-DRB1 alleles. Arthritis Rheum 2003;49:200-8.

20. Nyhall-Wahlin BM, Turesson C, Jacobsson L, Nilsson JX, Forslind K, Albertsson K, et al. The presence of rheumatoid nodules at early rheumatoid arthritis diagnosis is a sign of extraarticular disease and predicts radiographic progression of joint destruction over 5 years. Scand J Rheumatol. 2011;40:81-7

21. Nyhall-Wahlin BM, Jacobsson LT, Petersson IF, Turesson C. Smoking is a strong risk factor for rheumatoid nodules in early rheumatoid arthritis. Ann Rheum Dis 2006;65:601-6. 